\title{
Tricks for tract dilation study
}

\author{
Tevfik Ziypak · Senol Adanur · Turgut Yapanoglu
}

Received: 3 April 2014 / Accepted: 10 May 2014 / Published online: 23 May 2014

(C) Springer-Verlag Berlin Heidelberg 2014

\section{Dear Sir,}

We read the prospective randomized study comparing two percutaneous tract dilation methods (one-shot vs. telescopic dilation) with great interest [1]. So far, many researchers have done and published studies with the aim of finding the best dilation method for percutaneous nephrolithotomy (PNL) [2, 3]. We applaud the authors for doing a well-designed and conducted prospective study. However, some points of the study require clarification. When we read the Methodology section, we initially wondered how they estimated or calculated the number of patients (sample size) involved in each group. Did the authors aim to compare fluoroscopy time or other parameters, such as hemoglobin drop, success, or complication rate, between two techniques? The sample size may differ according to the parameter that the researchers want to analyze.

Fluoroscopy is the most commonly used imaging modality during endourologic surgeries. Retrograde pyelography, creation of access, tract dilation, evaluation of stone-free status, and antegrade pyelography are done using $\mathrm{C}$-arm fluoroscopy during PNL procedure. Therefore, the fluoroscopy time used for tract dilation phase or other phases should have been measured separately. In the study, the stone complexity, which affects fluoroscopic screening times, was similar in both groups; there is no information about mean stone size in each group. However, we think that the specific fluoroscopy time for tract dilation or tract creation could be a more significant parameter to compare both methods in terms of radiation safety.

The readers should want to know the exit strategies in the failure of one-shot tract dilation technique. We congratulate the study team that they achieved high success (94\%) for group-1, which included a high rate of staghorn and complex stone rate $(62 \%)$. While some intraoperative complications are described in the Results section, the comparison of overall complications, including postoperative ones, could be more informative for the readers.

Conflict of interest None.

\section{References}

1. Amirhassani S, Mousavi-Bahar SH, Iloon Kashkouli A, Torabian S (2014) Comparison of the safety and efficacy of one-shot and telescopic metal dilatation in percutaneous nephrolithotomy: a randomized controlled trial. Urolithiasis 42(3):269-273

2. Dehong C, Liangren L, Huawei L, Qiang W (2013) A comparison among four tract dilation methods of percutaneous nephrolithotomy: a systematic review and meta-analysis. Urolithiasis 41(6):523-530

3. Li Y, Yang L, Xu P, Shen P, Qian S, Wei W, Wang J (2013) Oneshot versus gradual dilation technique for tract creation in percutaneous nephrolithotomy: a systematic review and meta-analysis. Urolithiasis 41(5):443-448
T. Ziypak $(\bowtie) \cdot$ S. Adanur $\cdot$ T. Yapanoglu

Department of Urology, Medical Faculty, Ataturk University, Erzurum, Turkey

e-mail: tziypak@yahoo.com 